\title{
OBLIQUE INTERFACE-WAVE DIFFRACTION BY A SMALL BOTTOM DEFORMATION IN TWO SUPERPOSED FLUIDS
}

\author{
B. N. MANDAL \\ Physical and Earth Sciences Division \\ Indian Statıstıcal Instıtute \\ 203, Barrackpore Trunk Road \\ Calcutta 700035, INDIA \\ U. BASU \\ Department of Applied Mathematics \\ University of Calcutta \\ 92, A P C Road \\ Calcutta 700009, INDIA
}

(Received Augsut 5, 1993 and in revised form December 12, 1994)

\begin{abstract}
The problem of diffraction of oblique interface-waves by a small bottom deformation of the lower fluid in two superposed fluids has been investigated here assuming linear theory and invoking a simplified perturbation analysis First order corrections to the velocity potentials in the two fluids are obtained by using the Green's integral theorem in a suitable manner The transmission and reflection coefficients are evaluated approximately These reduce to the known results for a single fluid in the absence of the upper fluid
\end{abstract}

KEY WORDS AND PHRASES. Oblique interface-wave diffraction, transmission and reflection coefficients, water waves

1991 AMS SUBJECT CLASSIFICATION CODE. 76C10, 76D33

\section{INTRODUCTION.}

A time-harmonic progressive wave train propagating on the surface of an ocean experiences no reflection if the ocean is of uniform finite depth However, if the bottom of the ocean has a deformation, then the wave train is partially reflected by and partially transmitted over the bottom deformation Miles [1] obtained approximately the transmission and reflection coefficients for oblique surface-waves when the bottom has a small deformation in the form of a long cylinder in the lateral direction Mandal and Basu [2] extended this problem to include surface tension effect at the free surface

In the present paper the oblique surface-wave diffraction problem considered in [1] for a single fluid is generalized to two superposed fluids wherein the upper fluid extends infinitely upwards and the lower fluid is of finite but nonuniform depth below the mean interface and its bottom has a small deformation in the form of a long cylinder in the lateral direction Utilizing a simplified perturbational analysis directly to the governing partial differential equation and the boundary and other conditions describing the physical problem, the original boundary value problem (BVP) is reduced up to first order to another BVP Solution of this BVP is then obtained by an appropriate use of Green's integral theorem to the potential functions describing the BVP and source potentials given in [3] The first order corrections to the reflection and transmission coefficients are then evaluated from the requirements at infinity It is verified that in the absence of the upper fluid, known results for a single fluid are recovered 
It may be mentioned here that although the source potentials in each of two superposed fluids due to various types of basic singularities are known in the literature (cf [3],[4],[5]), their application in the study of interface-waves in two superposed fluids is rather limited In the present paper, the twodimensional source potentials in each of two superposed fluids obtained earlier in [3] have been used suitably in the Green's integral theorem to obtain representations for the first order corrections to the velocity potentials in each of the two fluids

\section{FORMULATION OF THE PROBLEM}

We consider two superposed immiscible, nonviscous, incompressible and homogeneous fluids with lower fluid of density $\rho_{1}$ occupying the region $0 \leq y \leq h+\epsilon c(x)$ and upper fluid of density $\rho_{2}\left(<\rho_{1}\right)$ occupying the region $y \leq 0$ Here, the plane $y=0$ is the position of the interface at rest and $y$-axis is taken vertically downwards into the lower fluid There is a small deformation at the bottom of the lower fluid in the lateral direction and is described by $y=h+\epsilon c(x)$ where $c(x)$ is a bounded and continuous function of compact support so that $c(x) \rightarrow 0$ as $|x| \rightarrow \infty$, and $\epsilon$ is a small positive number characterizing the smallness of the deformation Far away from the deformation, the lower fluid is of uniform finite depth $h$ The motion in each fluid is assumed to be small and irrotational so that it is described by the velocity potentials $\operatorname{Re}\left\{\Phi(x, y, z) e^{-\imath \sigma t}\right\}$ and $\operatorname{Re}\left\{\Psi(x, y, z) e^{-\imath \sigma t}\right\}$ in the lower and upper fluids respectively, $\sigma$ being the frequency of the incoming train of progressive waves at the interface and the time dependence $e^{-\imath \sigma t}$ being dropped throughout the analysis. Assuming linear theory, $\Phi$ and $\Psi$ satisfy the following coupled BVP.

$$
\begin{gathered}
\nabla_{1}^{2} \Phi=0 \quad \text { in the region } \quad 0 \leq y \leq h+\epsilon c(x), \\
\nabla_{1}^{2} \Psi=0 \quad \text { in the region } \quad y \leq 0
\end{gathered}
$$

where $\nabla_{1}^{2}$ is the three-dimensional Laplace operator,

$$
\begin{aligned}
\Phi_{y} & =\Psi_{y} \quad \text { on } \quad y=0, \\
K \Phi+\Phi_{y} & =s\left(K \Psi+\Psi_{y}\right) \quad \text { on } \quad y=0
\end{aligned}
$$

where $s=\rho_{2} / \rho_{1}, K=\sigma^{2} / g, g$ being the acceleration due to gravity,

$$
\Phi_{n}=0 \quad \text { on } \quad y=h+\epsilon c(x)
$$

where $n$ denotes the inward drawn normal to the bottom,

$$
\nabla{ }_{1} \Psi \rightarrow 0 \quad \text { as } \quad y \rightarrow \infty .
$$

A train of progressive interface-waves represented by the velocity potentials $\phi_{0}(x, y) e^{2 \nu z}$ and $\Psi_{0}(x, y) e^{i \nu z}$ in the lower and upper fluids respectively where

$$
\begin{gathered}
\phi_{0}(x, y)=\frac{\cosh \alpha(h-y)}{\sinh \alpha h} e^{\imath \mu x}, \\
\Psi_{0}(x, y)=-e^{\alpha y+\imath \mu x},
\end{gathered}
$$

is obliquely incident upon the bottom deformation from negative infinity. Here $\alpha$ is the unique positive zero of $\triangle(k)$ where

$$
\triangle(k)=K \cosh k h+\{s(K+k)-k\} \sinh k h,
$$

and $\nu=\alpha \sin \theta, \mu=\alpha \cos \theta$ where $\theta$ characterizes the oblique incidence of the wave train $\theta=0$ corresponds to normal incidence This wave train is partially reflected by and partially transmitted over the bottom deformation so that $\Phi$ and $\Psi$ satisfy the infinity requirements 


$$
\left[\begin{array}{l}
\Phi \\
\Psi
\end{array}\right] \sim\left\{\begin{array}{l}
T\left[\begin{array}{l}
\phi_{0}(x, y) \\
\psi_{0}(x, y)
\end{array}\right] e^{\prime \nu z} \text { as } \quad x \rightarrow \infty, \\
{\left[\begin{array}{l}
\phi_{0}(x, y) \\
\psi_{0}(x, y)
\end{array}\right] e^{\prime \nu z}+R\left[\begin{array}{l}
\phi_{0}(-x, y) \\
\psi_{0}(-x, y)
\end{array}\right] e^{\prime \nu z} \quad \text { as } \quad x \rightarrow-\infty,}
\end{array}\right.
$$

where $T$ and $R$ denote respectively the unknown transmission and reflection coefficients

Assuming $\epsilon$ to be very small, the bottom condition (2 5) can be expressed in approximate form [2] as

$$
-\Phi_{y^{\prime}}+\epsilon\left\{c^{\prime}(x) \Phi_{x}-c(x) \Phi_{y y}\right\}+0\left(\epsilon^{2}\right)=0 .
$$

In view of the geometry of the problems we can assume

$$
\begin{aligned}
& \Phi(x, y, z)=\phi(x, y) e^{\imath \nu z} \\
& \Psi(x, y, z)=\psi(x, y) e^{2 \nu z}
\end{aligned}
$$

so that $\phi(x, y)$ and $\psi(x, y)$ satisfy the following BVP

$$
\begin{array}{ccc}
\left(\nabla^{2}-\nu^{2}\right) \phi=0 \quad \text { in } & 0 \leq y \leq h+\epsilon c^{\prime}(x), \\
\left(\nabla^{2}-\nu^{2}\right) \psi=0 & \text { in } \quad y \leq 0
\end{array}
$$

where $\nabla^{2}$ is the two-dimensional Laplace operator,

$$
\begin{gathered}
\phi_{y}=\psi_{y} \quad \text { on } \quad y=0, \\
K \phi+\phi_{y}=s\left(K \psi+\psi_{y}\right) \quad \text { on } \quad y=0, \\
-\phi_{y}+\epsilon\left\{\frac{d}{d x}\left(c(x) \phi_{x}\right)-\nu^{2} \phi\right\}+\mathrm{O}\left(\epsilon^{2}\right)=0 \quad \text { on } \quad y=h
\end{gathered}
$$

since $\phi$ satisfies (2.14), and

$$
\nabla \psi \rightarrow 0 \quad \text { as } \quad y \rightarrow-\infty .
$$

Also $\phi, \psi$ satisfy the infinity requirements

$$
\left[\begin{array}{l}
\phi \\
\psi
\end{array}\right] \sim\left\{\begin{array}{r}
T\left[\begin{array}{l}
\phi_{0}(x, y) \\
\psi_{0}(x, y)
\end{array}\right] \quad \text { as } \quad x \rightarrow \infty \\
{\left[\begin{array}{l}
\phi_{0}(x, y) \\
\psi_{0}(x, y)
\end{array}\right]+R\left[\begin{array}{l}
\phi_{0}(-x, y) \\
\psi_{0}(-x, y)
\end{array}\right] \quad \text { as } \quad x \rightarrow-\infty}
\end{array}\right.
$$

\section{METHOD OF SOLUTION}

In view of the approximate bottom condition (2 18) coupled with the fact that an interface-wave train experiences no reflection if the lower fluid has a uniform bottom, we can assume a perturbation expansion for $\phi, \psi, T$ and $R$ in terms of $\epsilon$ as

$$
\begin{aligned}
& \phi=\phi_{0}+\epsilon \phi_{1}+\mathrm{O}\left(\epsilon^{2}\right), \\
& \psi=\psi_{0}+\epsilon \psi_{1}+\mathrm{O}\left(\epsilon^{2}\right), \\
& T=1+\epsilon T_{1}+\mathrm{O}\left(\epsilon^{2}\right), \\
& R=\epsilon R_{1}+\mathrm{O}\left(\epsilon^{2}\right) .
\end{aligned}
$$

Using the expansions (3.1) in (2 14) to (2 21) we find that $\phi_{1}, \psi_{1}$ satisfy the following BVP

$$
\left(\nabla^{2}-\nu^{2}\right) \phi_{1}=0 \quad \text { in } \quad 0 \leq y \leq h,
$$




$$
\begin{gathered}
\left(\nabla^{2}-\nu^{2}\right) \psi_{1}=0 \quad \text { in } \quad y \leq 0, \\
\phi_{1 y}=\psi_{1 y} \quad \text { on } \quad y=0, \\
K \phi_{1}+\phi_{1 y}=s\left(K \phi_{2}+\phi_{2 y}\right) \quad \text { on } \quad y=0, \\
\phi_{1 y}=q(x) \quad \text { on } \quad y=h
\end{gathered}
$$

where

$$
q(x)=\frac{1}{\sinh \alpha h}\left[\imath \mu \frac{d}{d x}\left(c(x) e^{\imath \mu x}\right)-\nu^{2} c(x) e^{\imath \mu x}\right]
$$

Also, $\phi_{1}, \psi_{1}$ satisfy the following infinity requirements,

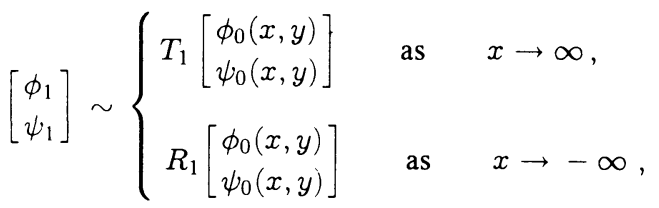

To solve the above coupled BVP, we need two-dimensional source potentials for the modified Helmholtz's equation due to a line source submerged in either of two superposed fluids wherein the lower fluid is of uniform finite depth below the mean interface $y=0$ and the upper fluid extends infinitely upwards When the source is submerged in the lower fluid at $(\xi, \eta)(0<\eta<h)$, let $G(x, y ; \xi, \eta)$ and $H(x, y ; \xi, \eta)$ denote the source potentials in the lower and upper fluids respectively, and when the source is submerged in the upper fluid at $(\xi, \eta)(\eta<0)$, let $\bar{G}(x, y ; \xi, \eta)$ and $\bar{H}(x, y ; \xi, \eta)$ denote the source potentials in the lower and upper fluids respectively Expressions for these source potentials and their asymptotic behaviors as $|x-\xi| \rightarrow \infty$ are given in [3] and are reproduced in the Appendix after correcting the misprints

To find $\phi_{1}(\xi, \eta)(0<\eta<h)$ we apply Green's integral theorem to $\phi_{1}(x, y)$ and $G(x, y ; \xi, \eta)$ in the region bounded externally by the lines $y=0(-X \leq x \leq X), \quad x= \pm X(0 \leq y \leq h)$, $y=h(-X \leq x \leq X)$ and internally by the circle $C$ with center at $(\xi, \eta)$ and radius $\delta$, and ultimately make $X \rightarrow \infty$ and $\delta \rightarrow 0$ We then obtain

$$
2 \pi \phi_{1}(\xi, \eta)=\int_{-\infty}^{\infty} q(x) G(x, h ; \xi, \eta) d x+\int_{-\infty}^{\infty}\left[\phi_{1} G_{y}-G \phi_{1 y}\right]_{y=0} d x
$$

Again, we apply the Green's integral theorem to $\psi_{1}(x, y)$ and $H(x, y ; \xi, \eta)$ in the region bounded externally by the lines $y=0(-X \leq x \leq X), x= \pm X(-Y \leq y \leq 0), y=Y(-X \leq x \leq X)$ and ultimately make $X, Y \rightarrow \infty$ Here we note that $H(x, y ; \xi, \eta)$ has no singularity in the region Then we find

$$
0=\int_{-\infty}^{\infty}\left[\psi_{1} H_{y}-H \psi_{1 y}\right]_{y=0} d x
$$

Multiplying ( $39 \mathrm{~b})$ by $s$ and subtracting from ( $39 \mathrm{a})$ we find

$$
2 \pi \phi_{1}(\xi, \eta)=\int_{-\infty}^{\infty} q(x) G(x, h ; \xi, \eta) d x+\int_{-\infty}^{\infty}\left[\left(\phi_{1} G_{y}-G \phi_{1 y}\right)-s\left(\psi_{1} H_{y}-H \psi_{1 y}\right)\right]_{y=0} d x
$$

Using the conditions ( 34 ) and ( 35 ) for $\phi_{1}$ and $\psi_{1}$ and the conditions on $y=0$ for $G$ and $H$ given in the Appendix, we find that on $y=0$, 


$$
\begin{aligned}
& \phi_{1} G_{y}-s \psi_{1} H_{y}=\left(\phi_{1}-s \psi_{1}\right) G_{y}=\frac{s-1}{K} \phi_{1 y} G_{y}, \\
& G \phi_{1 y}-s H \psi_{1 y}=(G-s H) \phi_{1 y}=\frac{s-1}{K} G_{y} \phi_{1 y} .
\end{aligned}
$$

Thus the term within the square bracket in the second integral vanishes identically Hence we obtain

$$
\phi_{1}(\xi, \eta)=\frac{1}{2 \pi} \int_{-\infty}^{\infty} q(x) G(x, h ; \xi, \eta) d x, \quad 0<\eta<h .
$$

To find $\psi_{1}(\xi, \eta)(\eta<0)$ we apply Green's integral theorem to $\psi_{1}(x, y)$ and $\bar{H}(x, y ; \xi, \eta)$ in the region bounded externally by the lines $y=0(-X \leq x \leq X), \quad x= \pm X(-Y \leq y \leq 0)$, $y=Y(-X \leq x \leq X)$ and internally by the circle $C^{\prime}$ of radius $\delta$ with center at $(\xi, \eta)$ and ultimately let $X, Y \rightarrow \infty$ and $\delta \rightarrow 0$ We then find

$$
2 \pi \psi_{1}(\xi, \eta)=-\int_{-\infty}^{\infty}\left[\psi_{1} \bar{H}_{y}-\bar{H} \psi_{1 y}\right]_{y=0} d x
$$

Again, we apply the Green's integral theorem to $\phi_{1}(x, y)$ and $\left.\bar{G} x, y ; \xi, \eta\right)(\eta<0)$ in the region bounded externally by the lines $y=0(-X \leq x \leq X), x= \pm X(0 \leq y \leq h), y=h(-X \leq x \leq X)$ and ultimately make $X \rightarrow \infty$ Noting that $\bar{G}$ has no singularity in the region we find

$$
0=\int_{-\infty}^{\infty}\left[\phi_{1} \bar{G}_{y}-\phi_{1 y} \bar{G}\right]_{y=0} d x+\int_{-\infty}^{\infty} q(x) \bar{G}(x, h ; \xi, \eta) d x
$$

Multiplying (3 11a) by $s$ and adding with (3 11b) we obtain

$$
2 \pi s \psi_{1}(\xi, \eta)=\int_{-\infty}^{\infty}\left[\left(\phi_{1} \bar{G}_{y}-\phi_{1 y} \bar{G}\right)-s\left(\psi_{1} \bar{H}_{y}-\psi_{1 y} \bar{H}\right)\right]_{y=0} d x+\int_{-\infty}^{\infty} q(x) \bar{G}(x, h ; \xi, \eta) d x
$$

The term in the square bracket of the second integral vanishes because of the conditions satisfied by $\phi_{1}, \psi_{1}$ and $\bar{G}, \bar{H}$ at $y=0$ Thus we find

$$
\psi_{1}(\xi, \eta)=\frac{1}{2 \pi s} \int_{-\infty}^{\infty} q(x) \bar{G}(x, h ; \xi, \eta) d x, \quad \eta<0 .
$$

\section{EVALUATION OF $T_{1}$ AND $R_{1}$}

$T_{1}$ and $R_{1}$ can be evaluated from the behavior of $\phi_{1}(\xi, \eta)$ or $\psi_{1}(\xi, \eta)$ as $\xi \rightarrow \infty$ and $-\infty$ respectively in (3.10) or (3 13). To find $T_{1}$ we note from (3.7) that

$$
\phi_{1}(\xi, \eta) \sim T_{1} \phi_{0}(\xi, \eta) \quad \text { as } \quad \xi \rightarrow \infty .
$$

Also from (3.10) after using (A3) we find as $\xi \rightarrow \infty$

$$
\phi_{1}(\xi, \eta) \sim\left[\frac{i \sinh \alpha h}{\mu\left(h+\frac{1-s}{K} \sinh ^{2} \alpha h\right)} \int_{-\infty}^{\infty} e^{-\imath \mu x} q(x) d x\right] \phi_{0}(\xi, \eta) .
$$

Thus

$$
\begin{aligned}
T_{1} & =\frac{i \sinh \alpha h}{\mu\left(+\frac{1-s}{K} \sinh ^{2} \alpha h\right)} \int_{-\infty}^{\infty} e^{-\imath \mu x} q(x) d x \\
& =-\frac{i \alpha \sec \theta}{h+\frac{1-s}{K} \sinh ^{2} \alpha h} \int_{-\infty}^{\infty} c(x) d x
\end{aligned}
$$

It is verified that the same expression for $T_{1}$ is also obtained by noting the behavior of $\psi_{1}(\xi, \eta)$ as $\xi \rightarrow \infty$ in $\left(\begin{array}{ll}3 & 7\end{array}\right)$ and $\left(\begin{array}{ll}3 & 13\end{array}\right)$.

Again, to obtain $R_{1}$, we note from (38) 


$$
\phi_{1}(\xi, \eta) \sim R_{1} \phi_{0}(-\xi, \eta) \quad \text { as } \quad \xi \rightarrow-\infty
$$

and using (A3) in (3 10) we find

$$
\phi_{1}(\xi, \eta) \sim\left[\frac{\imath \sinh \alpha h}{\mu\left(h+\frac{1-g}{K} \sinh ^{2} \alpha h\right)} \int_{-\infty}^{\infty} e^{\imath \mu x} q(x) d x\right] \phi_{0}(-\xi, \eta) \quad \text { as } \quad \xi \rightarrow-\infty .
$$

Thus

$$
\begin{aligned}
R_{1} & =\frac{i \sinh \alpha h}{\mu\left(h+\frac{1-s}{K} \sinh ^{2} \alpha h\right)} \int_{-\infty}^{\infty} e^{\imath \mu x} q(x) d x \\
& =\frac{i \alpha \sec \theta \cos 2 \theta}{h+\frac{1-s}{K} \sinh ^{2} \alpha h} \int_{-\infty}^{\infty} c(x) e^{2 \imath \mu x} d x
\end{aligned}
$$

It is again verified that the same expression for $R_{1}$ is also obtained by noting the behavior of $\psi_{1}(\xi, \eta)$ as $\xi \rightarrow-\infty$ in $\left(\begin{array}{ll}3 & 8\end{array}\right)$ and $\left(\begin{array}{ll}3 & 13\end{array}\right)$

It may be noted that in the absence of the upper fluid $(s=0)$, the results of [1] for a single fluid are recovered In that case $\alpha$ is the unique real positive zero of $\triangle(k)=K \cosh \alpha h-k \sinh k h$ The results for normal incidence of the wave train are obtained by putting $\theta=0$ For $\theta=\pi / 4, R_{1}$ vanishes independently of the bottom deformation This was also observed by Miles [1] for a single fluid Also, once the functional form of $c(x)$ is known, $T_{1}$ and $R_{1}$ can be obtained explicitly

\section{APPENDIX}

(a) $G(x, y ; \xi, \eta)$ and $H(x, y ; \xi, \eta)$

$$
\begin{aligned}
& G(x, y ; \xi, \eta) \text { and } H(x, y ; \xi, \eta) \text { satisfy } \\
& \left(\nabla^{2}-\nu^{2}\right) G=0 \text { in } 0 \leq y \leq h \text { except at }(\xi, \eta)(0<\eta<h), \\
& G \sim K_{0}(\nu r) \text { as } r=\left\{(x-\xi)^{2}+(y-\eta)^{2}\right\}^{1 / 2} \rightarrow 0, \\
& \left(\nabla^{2}-\nu^{2}\right) H=0 \text { in } y \leq 0, \\
& G_{y}=H_{y} \text { on } y=0, \\
& K G+G_{y}=s\left(K H+H_{y}\right) \text { on } y=0, \\
& G_{y}=0 \text { on } y=h, \quad \nabla H \rightarrow 0 \text { as } y \rightarrow-\infty,
\end{aligned}
$$

$G, H$ have outgoing nature as $|x-\xi| \rightarrow \infty$. Then $G(x, y ; \xi, \eta)$ and $H(x, y ; \xi, \eta)$ are given by (cf [3]) after correcting some misprints

$$
\begin{aligned}
G(x, y ; \xi, \eta) & =K_{0}(\nu r)-\frac{1-s}{1+s} K_{0}\left(\nu r^{\prime}\right) \\
& +\frac{2}{1+s} \int_{\nu}^{\infty}\left[\frac{e^{-k h}\{s(K+k)-k\}(\sinh k \eta+s \cosh k \eta) \operatorname{sech} k h-k(1-s) e^{-k \eta}}{\triangle(k)}\right. \\
& \left.\times \cosh k(h-y)+\frac{e^{-k h}(\sinh k \eta+s \cosh k \eta)}{\cosh k h} \sinh k y\right] \frac{\cos \left\{\left(k^{2}-\nu^{2}\right)^{1 / 2}(x-\xi)\right\}}{\left(k^{2}-\nu^{2}\right)^{1 / 2}} d k \\
H(x, y ; \xi, \eta) & =\frac{2}{1+s} K_{0}(\nu r)+\frac{2}{1+s} \int_{\nu}^{\infty}\left[\frac{\sinh k \eta+s \cosh k \eta}{\cosh k h} e^{-k h}\right. \\
& \left.-\frac{e^{-k h}\{s(K+k)-k\}(\sinh k \eta+s \cosh k \eta) \operatorname{sech} k h-k(1-s) e^{-k \eta}}{\triangle(k)} \sinh k h\right] \\
& \times e^{k y} \frac{\cos \left\{\left(k^{2}-\nu^{2}\right)^{1 / 2}(x-\xi)\right\}}{\left(k^{2}-\nu^{2}\right)^{1 / 2}} d k
\end{aligned}
$$


where $r^{\prime}=\left\{(x-\xi)^{2}+(y+\eta)^{2}\right\}^{1 \cdot 2}$ and the contour in each integral is indented below the pole at $k=\alpha$ to ensure the outgoing behavior of $G$ and $H$ as $|x-\xi| \rightarrow \infty \quad$ From (A1) and (A2) it can be shown that as $|x-\xi| \rightarrow \infty$

$$
\begin{aligned}
& G(x, y ; \xi, \eta) \sim 2 \pi i \frac{\cosh \alpha(h-\eta) \cosh \alpha(h-y)}{h+\frac{1}{h} \sinh \alpha h} \frac{e^{\prime n^{2} \nu^{\prime \prime}{ }^{\prime}, \leqslant}}{\left(\alpha^{2}-\nu^{2}\right)^{12}}, \\
& H(x, y ; \xi, \eta) \sim-2 \pi \imath \frac{\cosh \alpha(h-\eta) \sinh \alpha h e^{\alpha y}}{h+\frac{1 \cdot-g}{K} \sinh \alpha h} \frac{e^{\left.\iota, \alpha^{2}-\nu^{2}\right)^{1 / 2} \mid x-\xi}}{\left(\alpha^{2}-\nu^{2}\right)^{1 / 2}}
\end{aligned}
$$

(b) $\bar{G}(x, y ; \xi, \eta)$ and $\bar{H}(x, y ; \xi, \eta)$

$$
\begin{aligned}
& \bar{G}(x, y ; \xi, \eta) \text { and } \bar{H}(x, y ; \xi, \eta) \text { satisfy } \\
& \left(\nabla^{2}-\nu^{2}\right) \bar{G}=0 \text { in } 0 \leq y \leq h, \\
& \left(\nabla^{2}-\nu^{2}\right) \bar{H}=0 \text { in } y \leq 0 \text { except at }(\xi, \eta)(\eta<0), \\
& \bar{H} \sim K_{0}(\nu r) \text { as } r \rightarrow 0, \\
& \bar{G}_{y}=\bar{H}_{y} \text { on } y=0, \\
& K \bar{G}+\bar{G}_{y}=s\left(K \bar{H}+\bar{H}_{y}\right) \text { on } y=0, \\
& \bar{G}_{y}=0 \text { on } y=h, \quad \nabla \bar{H} \rightarrow 0 \text { as } y \rightarrow-\infty,
\end{aligned}
$$

$\bar{G}, \bar{H}$ have outgoing nature as $|x-\xi| \infty$ Then $G(x, y ; \xi, \eta)$ and $\bar{H}(x, y ; \xi, \eta)$ are given by (cf [3]) after correcting the misprints

$$
\begin{gathered}
\bar{G}(x, y ; \xi, \eta)=\frac{2 s}{1+s}\left[K_{0}(\nu r)+\int_{\nu}^{\infty}\left\{\frac{(1-s) k+\{s(K+k)-k\} e^{-k h} \operatorname{sech} k h}{\triangle(k)} \cosh k(h-y)\right.\right. \\
\left.\left.+\frac{e^{-k h}}{\cosh k h} \sinh k y\right\} e^{k \eta} \frac{\cos \left\{\left(k^{2}-\nu^{2}\right)^{1 / 2}(x-\xi)\right\}}{\left(k^{2}-\nu^{2}\right)^{1 / 2}} d k\right] \\
\bar{H}(x, y ; \xi, \eta)= \\
\quad K_{0}(\nu r)+\frac{1-s}{1+s} K_{0}\left(\nu r^{\prime}\right)+\frac{2 s}{1+s} \int_{\nu}^{\infty}\left[e^{-k h} \operatorname{sech} k h\right. \\
\left.-\frac{(1-s) k+s(K+k)-k e^{-k h} \operatorname{sech} k h}{\triangle(k)} \sinh k h\right] \\
\times e^{k(y+\eta)} \frac{\cos \left\{\left(k^{2}-\nu^{2}\right)^{1 / 2}(x-\xi)\right\}}{\left(k^{2}-\nu^{2}\right)^{1 / 2}} d k
\end{gathered}
$$

where again the contour in each integral is indented below the pole $k=\alpha$ to ensure the outgoing behavior of $\bar{G}$ and $\bar{H}$ as $|x-\xi| \rightarrow \infty$. From (A5) and (A6) it can be shown that as $|x-\xi| \rightarrow \infty$

$$
\begin{gathered}
\bar{G}(x, y ; \xi, \eta) \sim-2 s \pi i \frac{e^{\alpha \eta} \sinh \alpha h \cosh \alpha(h-y)}{h+\frac{1-s}{K} \sinh ^{2} \alpha} \frac{e^{i\left(\alpha^{2}-\nu^{2}\right)^{1 / 2}|x-\xi|}}{\left(\alpha^{2}-\nu^{2}\right)^{1 / 2}}, \\
\bar{H}(x, y ; \xi, \eta) \sim 2 s \pi i \frac{e^{\alpha(\eta+y)} \sinh ^{2} \alpha h}{h+\frac{1-s}{K} \sinh ^{2} \alpha h} \frac{e^{2\left(\alpha^{2}-\nu^{2}\right)^{1 / 2}|x-\xi|}}{\left(\alpha^{2}-\nu^{2}\right)^{1 / 2}}
\end{gathered}
$$




\section{REFERENCES}

[1] MILES, J W, Oblique surface wave diffraction by a cylindrical obstacle, Dyn. Atmos. Oceans 6 (1981), 121-123

[2] MANDAL, BN and BASU, U, A note on oblique water wave diffraction by a cylindrical deformation of the bottom in the presence of surface tension, Arch. Mech. 42 (1990), 723-727

[3] MANDAL, B N and CHAKRABARTI, R N, Two-dimensional, source potentials in a two-fluid medium for the modified Helmholtz's equation, Internat. J. Math. \& Math. Scl. 9 (1986), 175-184

[4] GORGUI, M A and KASSEM, S A, Basic singularities in the theory of internal waves, $Q . J l$. Mech. Appl. Math. 31 (1978), 31-48.

[5] RHODES-ROBINSON, P F , On waves at an interface between two liquids, Math. Proc. Camb. Phil. Soc. 38 (1980), 183-191 


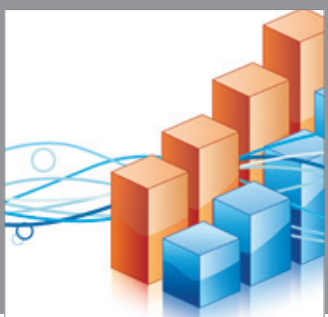

Advances in

Operations Research

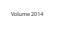

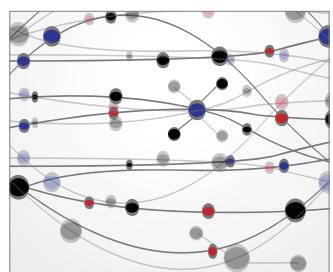

\section{The Scientific} World Journal
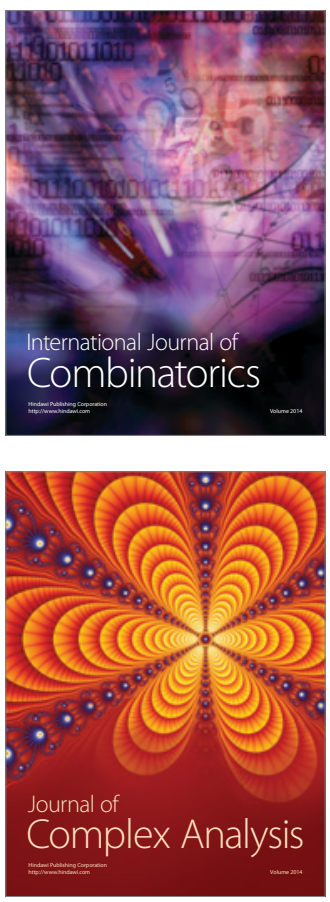

International Journal of

Mathematics and

Mathematical

Sciences
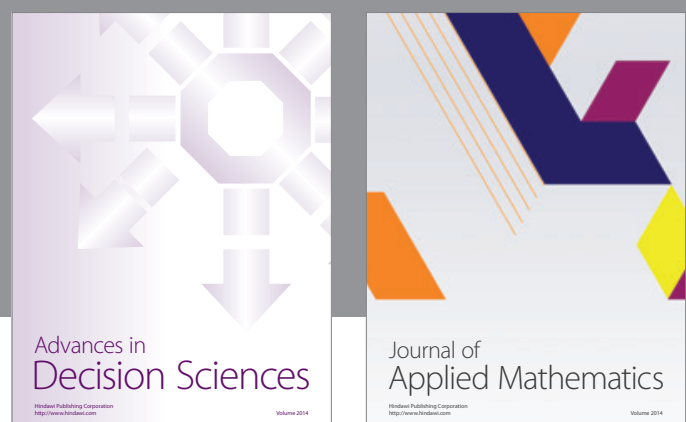

Journal of

Applied Mathematics
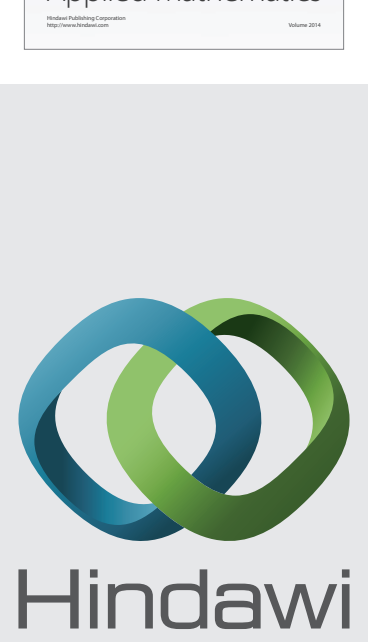

Submit your manuscripts at http://www.hindawi.com
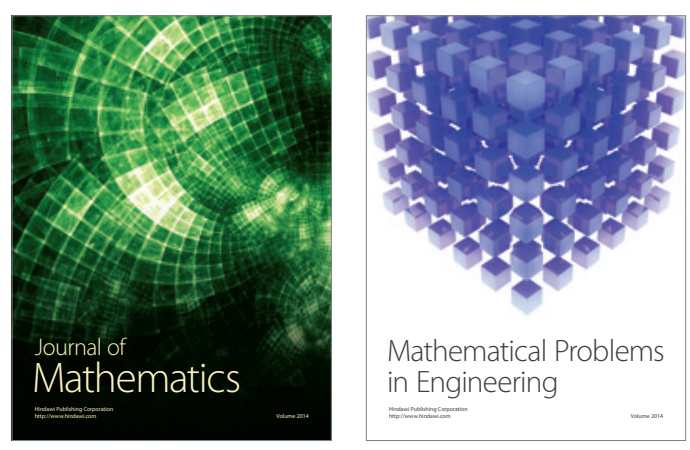

Mathematical Problems in Engineering
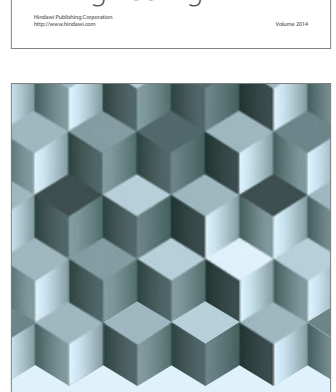

Journal of

Function Spaces
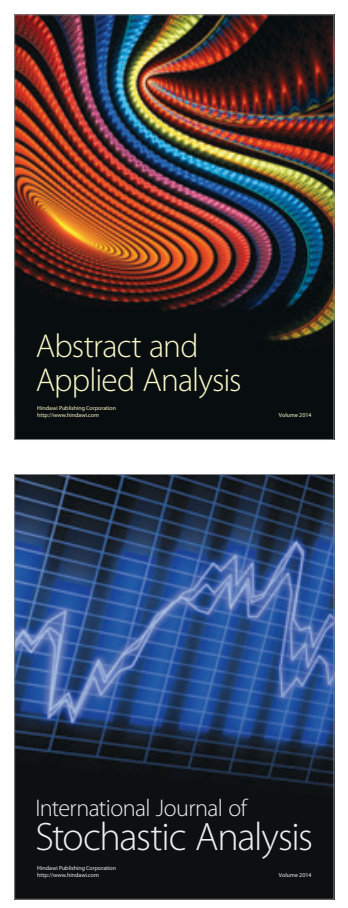

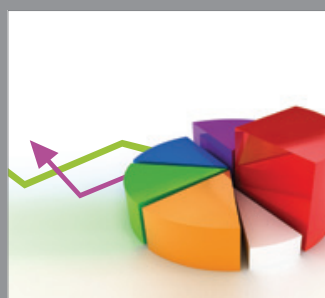

ournal of

Probability and Statistics

Promensencen
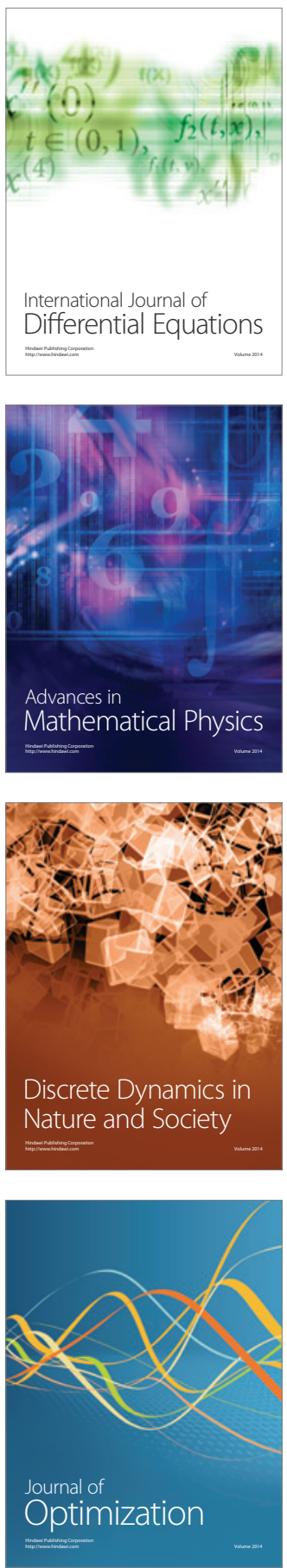